\title{
Multielement Synthetic Transmit Aperture Imaging Using Temporal Encoding
}

\section{Gammelmark, Kim; Jensen, Jørgen Arendt}

\section{Published in:}

I E E E Transactions on Medical Imaging

Link to article, DOI:

10.1109/TMI.2003.809088

Publication date:

2003

Document Version

Publisher's PDF, also known as Version of record

Link back to DTU Orbit

Citation (APA):

Gammelmark, K., \& Jensen, J. A. (2003). Multielement Synthetic Transmit Aperture Imaging Using Temporal Encoding. I E E E Transactions on Medical Imaging, 22(4), 552-563. https://doi.org/10.1109/TMI.2003.809088

\section{General rights}

Copyright and moral rights for the publications made accessible in the public portal are retained by the authors and/or other copyright owners and it is a condition of accessing publications that users recognise and abide by the legal requirements associated with these rights.

- Users may download and print one copy of any publication from the public portal for the purpose of private study or research.

- You may not further distribute the material or use it for any profit-making activity or commercial gain

- You may freely distribute the URL identifying the publication in the public portal

If you believe that this document breaches copyright please contact us providing details, and we will remove access to the work immediately and investigate your claim 


\title{
Multielement Synthetic Transmit Aperture Imaging Using Temporal Encoding
}

\author{
Kim Løkke Gammelmark, Student Member, IEEE, and Jørgen Arendt Jensen, Senior Member, IEEE
}

\begin{abstract}
A new method to increase the signal-to-noise ratio (SNR) of synthetic transmit aperture imaging is investigated. The approach utilizes multiple elements to emulate a spherical wave, and the conventional short excitation pulse is replaced by a linear frequency-modulated (FM) signal. The approach is evaluated in terms of image quality parameters in comparison to linear array imaging. Field II simulations using an 8.5-MHz linear array transducer with 128 elements show an improvement in lateral resolution of up to $30 \%$ and up to $10.75 \%$ improvement in contrast resolution for the new approach. Measurements are performed using our experimental multichannel ultrasound scanning system, RASMUS. The designed linear FM signal obtains temporal sidelobes below $\mathbf{- 5 5} \mathrm{dB}$, and SNR investigations show improvements of 4-12 dB. A $30 \mathrm{~mm}(\approx 45 \%)$ increase in penetration depth is obtained on a multitarget phantom with $0.5 \mathrm{~dB} /[\mathrm{cm} \mathrm{MHz}]$ attenuation. Furthermore, in vivo images of the abdomen are presented, which demonstrate the clinical application of the new approach.
\end{abstract}

Index Terms-Experimental ultrasound scanning system, Field II, linear FM signals, multielement defocusing, synthetic transmit aperture ultrasound imaging.

\section{INTRODUCTION}

$\mathbf{O}$ NE OF the main problems in ultrasound imaging is the tradeoff between penetration depth and spatial resolution. The resolution is improved as the frequency increases, but the attenuation in soft tissue also increases with increasing frequency. Thus, to increase the penetration depth while the resolution is maintained, the transmitted energy must be increased. This can be done by increasing the amplitude and/or the duration of the excitation pulse.

The United States Food and Drug Administration (US FDA) has introduced preamendments upper limits on the acoustic output of an ultrasound scanner [1], to avoid damage to the tissue and pain to the patient. These limits concern the mechanical index (MI), the derated spatial-peak-temporal-average intensity $\left(I_{\text {spta.3 }}\right)$, and the derated spatial-peak-pulse-average intensity $\left(I_{\text {sppa.3 }}\right)^{1}[1]$, [2]. MI is proportional to derated peak

Manuscript received August 9, 2002; revised November 4, 2002. This work was supported in part by the Danish Science Foundation under Grant 9700883 and Grant 9700563, in part by Technical University of Denmark under Ph.D. Grant 11-3160-55173, and in part by B-K Medical A/S, Herlev, Denmark. The Associate Editor responsible for coordinating the review of this paper and recommending its publication was M. Insana. Asterisk indicates corresponding author.

*K. L. Gammelmark is with the Center for Fast Ultrasound Imaging, Ørsted - DTU, Building 348, Technical University of Denmark, 2800 Kgs. Lyngby, Denmark (e-mail: klg@oersted.dtu.dk).

J. A. Jensen is with the Center for Fast Ultrasound Imaging, Ørsted • DTU, Technical University of Denmark, 2800 Kgs. Lyngby, Denmark.

Digital Object Identifier 10.1109/TMI.2003.809088

${ }^{1}$ The thermal index (TI) has not been included in the analysis in this paper. rarefraction pressure [3], while $I_{\mathrm{spta} .3}$ and $I_{\mathrm{sppa} .3}$ depend both on pulse shape and duration [2].

The peak pressure in the tissue is proportional to the peak amplitude of the excitation pulse. For the conventional imaging techniques like linear array imaging the peak amplitude of the short excitation pulses used can, therefore, not be increased to overcome the loss in penetration depth, when high frequencies are used. This is because the $I_{\text {sppa.3 }}$ limit is reached much earlier than the $I_{\text {spta.3 }}$ limit for these systems due to the transmit focusing. For short pulse systems in general, the $I_{\text {sppa.3 }}$ limit is very often reached much earlier than the $I_{\text {spta.3 }}$ limit, which limits the amplitude of the excitation pulse. Thus, to increase the penetration depth, the temporal energy and, thus, $I_{\text {spta.3 }}$, must be increased. This is done by increasing the duration of the transmitted pulse. For this purpose, the conventional pulse cannot be used, since the axial resolution is inversely proportional to the bandwidth (after matched filtering). Thus, some type of temporal encoding is necessary. This requirement is satisfied by linear frequency-modulated (FM) signals [4], [5], which have been used in radar systems for decades, because of their compression ability. These signals have recently been applied in ultrasound scanners [6]-[10], and they have shown good performance in terms of increased penetration depth, while maintaining the spatial resolution.

Generally, linear array imaging systems obtain a good spatial resolution due to the application of dynamic receive focusing. The resolution can, however, be improved by increasing the number of transmit foci. This is often done in modern ultrasound scanners at the expense of a reduction in the frame rate proportional to the number of foci. One way to obtain high spatial resolution, while keeping the frame rate high, is to use the synthetic transmit aperture (STA) imaging technique [11]. The inherent nature of this technique makes it possible to generate images with dynamic transmit and receive focusing using only a few transmissions. Therefore, STA imaging offers very high frame rates, which makes it suitable for real-time three-dimensional (3-D) volumetric imaging. A problem in STA imaging is, however, the low signal-to-noise ratio (SNR), due to the application of a single transmit element. This means that the penetration depth obtained by STA imaging is much lower than that obtained by linear array imaging, which significantly limits its clinical application. However, if the SNR can be increased to that of linear array imaging, images with better image quality can be generated, while maintaining a high frame rate. For this purpose the linear FM signal can be applied with great advantage. Also, previous research has shown [11], [12] that the application of multielement subapertures in STA imaging to produce a spherical wave transmission yields spatial resolution results 
very close to the single element transmission, and also increases the SNR proportionally to the number of elements used in the subaperture. Another potential problem in STA imaging is tissue motion. Although this issue is not considered in this paper, an example of its influence in abdominal imaging is given in Section $\mathrm{V}$ using measured in vivo tissue velocities. The example shows, that the effects of tissue motion on the image quality are minor, and this is supported by presented abdominal in vivo images.

This paper investigates the combination of these two methods to increase the SNR of STA imaging. The approach utilizes a multielement subaperture to produce a spherical wave with high power, and the conventional short excitation signal is replaced by a linear FM signal. Initially, the principles of classical STA imaging is presented in Section II along with STA beamforming. Section III describes the new approach in detail and gives measures of the expected increase in SNR. Section IV presents simulation results using Field II, and Section V presents phantom and in vivo measurement results of the abdomen using our experimental multichannel ultrasound scanning system, RASMUS [13]. All results are compared to corresponding linear array imaging results. The paper is concluded in Section VI.

\section{Synthetic Transmit APERTURE IMAging}

In conventional STA imaging a single element is used at each transmission to produce a spherical wave that interrogates a large region of the medium (see Fig. 1). The echoes resulting from scattering in the medium are recorded using all elements in the aperture, and the procedure is repeated until a desired number of elements have been used for transmission. For each transmission the echoes received by each element in the aperture contain information about all scatterers in the interrogated region. Since no focusing has been applied on transmit, and the exact origin of the spherical wave is known, the receive aperture can be steered in any direction and focused at any point within this region. Therefore, every point in the image can be formed independent of each other, and a full image is obtained for every transmission event with low lateral resolution due to the single transmit element. Taking into account the full path length between the transmitting element and the receive elements, when calculating the beamforming delays (as illustrated in Fig. 2), the individual low resolution images are both dynamically focused on receive, and adjusted to obtain dynamic focusing on transmit in the same operation. Dynamic focusing on transmit is then obtained subsequently by coherently summing the low resolution images which forms the final high resolution image as illustrated in Fig. 1.

\section{A. STA Beamforming}

This section describes how STA beamforming is performed and the flexibility in a STA beamformer.

Fig. 2 shows the concept of STA beamforming from a spatial point of view. The image point to be beamformed is described by the vector $\vec{r}_{p}$, and the vectors $\vec{r}_{m}$ and $\vec{r}_{n}$ designate the position of transmit element $m$ and receive element $n$, respectively. Letting $g_{m, n}(t)$ represent the received echo signal on element

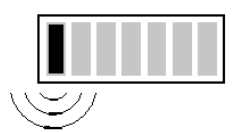

Transmit aperture

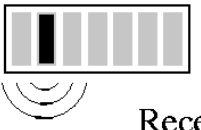

Receive aperture
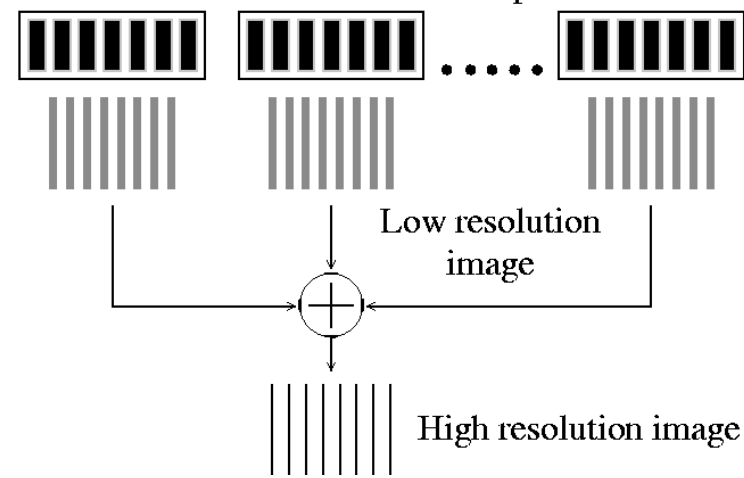

Fig. 1. Illustration of the conventional STA imaging principle. At each transmission event a single element is fired, and the echoes from the medium are collected using all elements in the receiving aperture. For each of these transmission events a complete image is beamformed, and these are subsequently summed to form the final high resolution image.

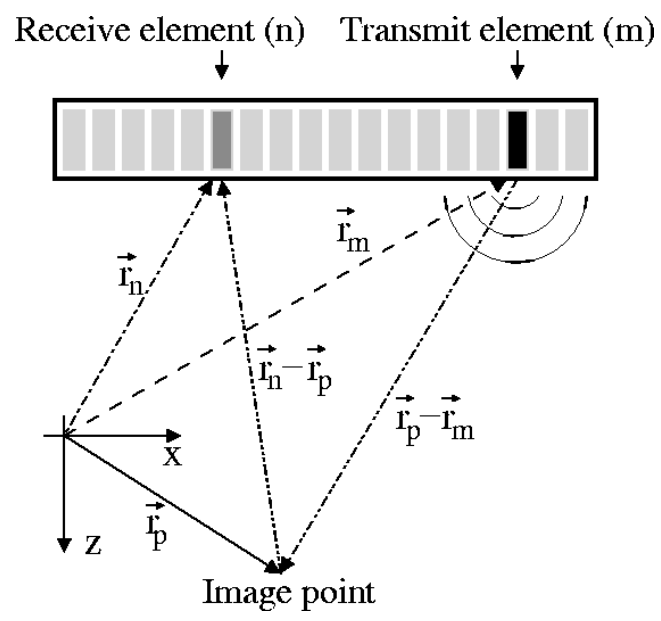

Fig. 2. Illustration of STA beamforming from a spatial view point. The time delay used for picking out the correct sample, in order to obtain transmit and receive focusing at the illustrated image point, is determined by the absolute distance between the image point and the transmit and receive elements, $m$ and $n$, respectively.

$n$ when transmitting with element $m$, then the low resolution image, $s_{m}\left(\vec{r}_{p}\right)$, for transmit element $m$ is calculated by

$$
s_{m}\left(\vec{r}_{p}\right)=\sum_{n=1}^{N} w_{n}\left(\vec{r}_{p}\right) g_{m, n}\left(\frac{d_{m, n}\left(\vec{r}_{p}\right)}{c}\right)
$$

where $w_{n}\left(\vec{r}_{p}\right)$ is the apodization values for receive element $n$, $c$ is the sound speed, and $N$ is the number of receive channels. The total distance $d_{m, n}\left(\vec{r}_{p}\right)$ from the transmitting element to the image point and back to the receiving element is given by

$$
d_{m, n}\left(\vec{r}_{p}\right)=\left|\vec{r}_{p}-\vec{r}_{m}\right|+\left|\vec{r}_{n}-\vec{r}_{p}\right|
$$

where the individual distances are defined in Fig. 2. This distance describes the time at which the scattered signal from the image point is received at element $n$ when transmitting with element $m$. Keeping the transmit element fixed, $d_{m, n}\left(\vec{r}_{p}\right)$ determines which samples should be picked out of the received signals in order to obtain a receive focus at the image point. However, keeping the receive element fixed and letting the transmit 
element move, $d_{m, n}\left(\vec{r}_{p}\right)$ also determines the time shift between the received signals from different transmissions and, thus, the shift between the low resolution images. Therefore, when applying $d_{m, n}\left(\vec{r}_{p}\right)$ in (1) both dynamic receive focusing is obtained and $s_{m}\left(\vec{r}_{p}\right)$ is adjusted such that dynamic transmit focusing is obtained, when the low resolution images are subsequently summed. Thus, the final high resolution image, $S\left(\vec{r}_{p}\right)$, with both dynamic transmit and receive focusing, is obtained by summing the low resolution images (neglecting motion compensation). In particular

$$
S\left(\vec{r}_{p}\right)=\sum_{m=1}^{M} w_{m}\left(\vec{r}_{p}\right) s_{m}\left(\vec{r}_{p}\right)
$$

where $w_{m}\left(\vec{r}_{p}\right)$ is the apodization value for transmit element $m$. In general, the time at which the sample is to be picked out of the received signals, $g_{m, n}(t)$, does not correspond to an integer sample number. Therefore, interpolation is needed to find the correct sample value. If interpolation is not used, a high sampling frequency is necessary in order to limit the increase in sidelobe and grating lobe levels, due to the phase quantization [14]. An alternative to using interpolation, which can be hardware demanding, is to use phase rotation in the digital beamformer as suggested by O'Donnell and coworkers in [15].

The advantage of the above beamforming scheme is that only one interpolation operation is needed to obtain $S\left(\vec{r}_{p}\right)$ compared to normally two, when transmit and receive focusing is separated. This is both cost and time efficient and reduces interpolation artifacts.

The transmit and receive apodization values can be used to generate any type of dynamic aperture and dynamic apodization function in order to obtain the desired beam shape. The dynamic aperture is typically selected to grow linearly, while keeping a constant F-number. This ensures a constant beam width until the aperture is fully opened. The shape of the dynamic apodization function determines the tradeoff between sidelobe levels and lateral resolution. Note that any point in the image can be beamformed independently of all other image points using the above scheme.

\section{Temporally ENCODED Multielement (TMS) STA IMAGING}

The objective of this paper is to investigate a new approach to increase the SNR of conventional STA imaging. It is called temporally encoded multielement STA (TMS) imaging, and it is based on the combination of multielement STA imaging with linear FM waveforms. These two techniques are described briefly in the following.

\section{A. Multielement STA Imaging}

The major drawback of STA imaging is the low SNR. Since a single element is used at each transmission the SNR is very low compared to, e.g., linear array imaging, which significantly limits its clinical application. To overcome this, a subaperture consisting of multiple grouped elements can be used to emulate the radiation pattern of a (virtual) high power point source, i.e., a spherical wave, and hereby increase the SNR. This concept is called multielement synthetic transmit aperture imaging, and it has previously been investigated by Karaman and coworkers

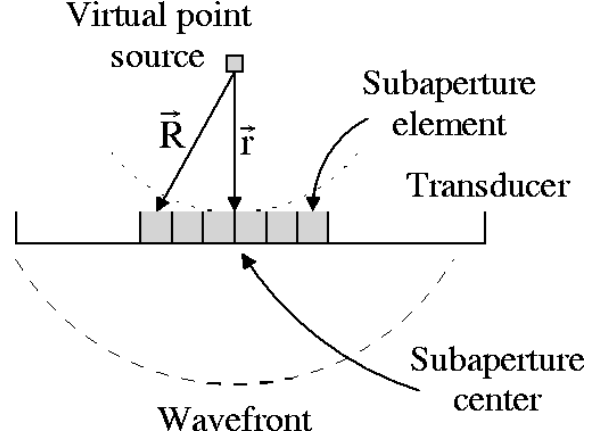

Fig. 3. Illustration of the setup used when calculating the transmit delays for emulating a spherical wave emitted from a virtual high power point source positioned behind the subaperture.

[11] with respect to application in hand-held scanners, and by Lockwood and coworkers [16] with the purpose of 3-D ultrasound imaging. Both groups show that by properly delaying the individual elements in the subaperture, good approximation to a spherical wave can be obtained along with a significant improvement in SNR.

The defocusing delay for each subaperture element can be calculated as illustrated in Fig. 3. The position of a virtual point source is selected behind the center of the subaperture with a certain axial distance selected, e.g., as a fraction of the subaperture size. Given the subaperture elements coordinates and the center of the subaperture, the defocusing delay $\tau_{d}$ for a specific element is then calculated by

$$
\tau_{d}=\frac{|\vec{R}|-|\vec{r}|}{c}
$$

where $\vec{R}$ is the vector from the virtual point source to the element, and $\vec{r}$ is the vector from the virtual point source to the subaperture center.

The position of the virtual point source, which produces the best emulation of the spherical wave, is not straight forward to calculate, because it depends on the interference between the waves from the individual subaperture elements and their radiation patterns. If the virtual source is moved away from the subaperture, the beam will become increasingly flat and narrow, and at some point yield a nearly plane wave for the given subaperture. The virtual element cannot be moved too close to the subaperture either, because the difference between the transmit delays becomes too large to obtain a coherent wavefront. Therefore, the proper location of the virtual point source should be investigated through simulations. These results are presented in Section IV.

\section{B. Temporal Encoding Using Linear FM Signals}

Linear FM signals have been used in radars for decades because of their distinctive feature of serving as both a long emitted and a short compressed pulse simultaneously. In medical ultrasound imaging, one of the first frameworks for using coded signals to improve the penetration-resolution problem was described by Takeuchi in 1979 [6]. Since then several authors have investigated different approaches and schemes to fully utilize their capabilities and improve their performance (e.g., [7], [10], and [17]-[19]). 

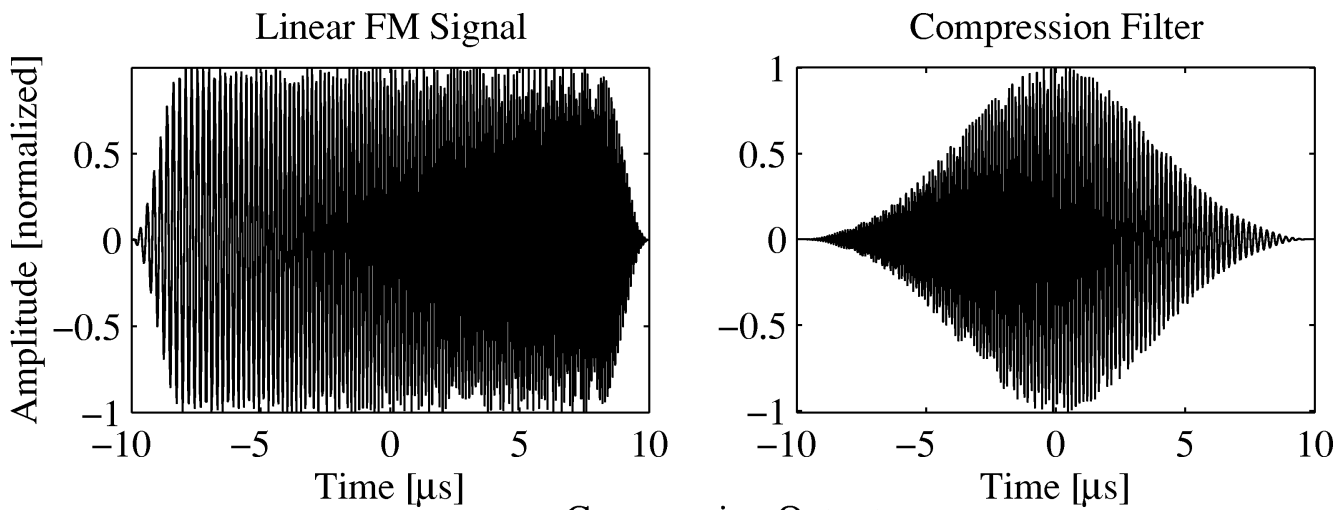

Compression Output

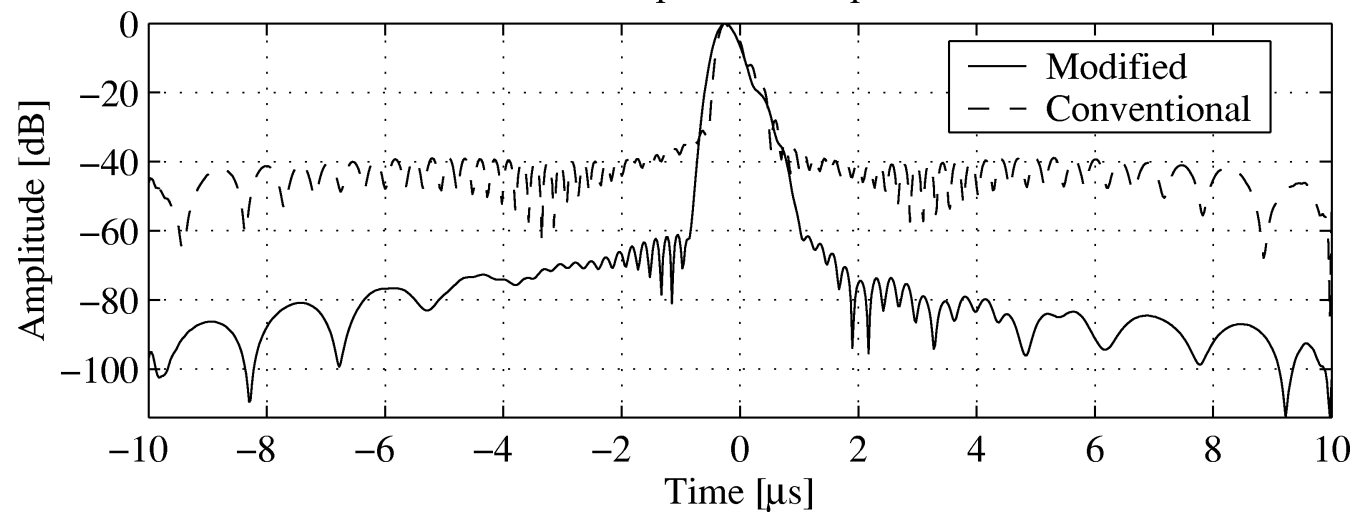

Fig. 4. Top left: Modified linear FM signal with a 7-MHz center frequency and 7-MHz bandwidth. A Tukey window with a duration of $10 \%$ has been applied. Top right: Modified compression filter using a Chebychev window with $70 \mathrm{~dB}$ relative sidelobe attenuation. Bottom: Compression output for the conventional FM signal (dashed) and the modified FM signal (solid). The effect of a linear array transducer has been introduced in the compression outputs.

The conventional linear FM signal is described in [4] as

$$
p(t)=b \cos \left(2 \pi\left[f_{c} t+\frac{B_{s}}{2 \tau_{s}} t^{2}\right]\right), \quad|t| \leq \frac{\tau_{s}}{2}
$$

where $b$ is the amplitude, $f_{c}$ is the center frequency, $B_{s}$ is the frequency sweep (the bandwidth), and $\tau_{s}$ is the duration of the signal. The matched filter to $p(t)$ in (5) is given by

$$
\begin{aligned}
h_{m}(t) & =p(-t) \\
& =b \cos \left(2 \pi\left[f_{c} t-\frac{B_{s}}{2 \tau_{s}} t^{2}\right]\right), \quad|t| \leq \frac{\tau_{s}}{2} .
\end{aligned}
$$

Assuming $p(t)$ is the waveform received from a point target, the compressed radio-frequency signal becomes [4]

$$
\begin{aligned}
g(t) & =p(t) * h_{m}(t) \\
& =\frac{b^{2} \tau_{s}}{2} \frac{\sin \left(\pi D \frac{t}{\tau_{s}}\left(1-\frac{|t|}{\tau_{s}}\right)\right)}{\pi D \frac{t}{\tau_{s}}} \cos \left(2 \pi f_{c} t\right)
\end{aligned}
$$

where $D=\tau_{s} B_{s}$ is the time-bandwidth product, $|t| \leq \tau_{s}$, and higher frequency terms have been neglected. Considering only the envelope of $g(t)$, this has approximately the shape of a sinc-function. Assuming $D$ is large (generally above 20 as mentioned by Blinchikoff and Zverev [4]), the envelope of $g(t)$, $\hat{g}(t)$, becomes a sinc-function

$$
\hat{g}(t) \approx|g(t)| \approx \frac{b^{2} \tau_{s}}{2} \frac{\sin \left(\pi D \frac{t}{\tau_{s}}\right)}{\pi D \frac{t}{\tau_{s}}}, \quad|t| \leq \tau_{s} .
$$

The $2 / \pi \approx-4 \mathrm{~dB}$ width of this function is $\delta t=1 / B_{s}$, which shows that the temporal (axial) resolution can be improved by increasing the bandwidth. This is advantageous when using high bandwidth transducers.

An example of the compression result in (8) is shown as the dashed curve in Fig. 4 (bottom). The influence of an $8.5-\mathrm{MHz}$ linear array transducer has been introduced, and the parameters for $p(t)$ and $h_{m}(t)$ are $f_{c}=7 \mathrm{MHz}, B_{s} \simeq 7 \mathrm{MHz}$, and $\tau_{s}=20$ $\mu \mathrm{s}$. As seen the temporal sidelobes are around $-40 \mathrm{~dB}$, which is not sufficient for medical imaging. To reduce the sidelobes, previous research has shown that this can effectively be done by applying amplitude weighting on the linear FM signal and compression filter [8], [19], [20]. In this study, a Tukey window with a $10 \%$ duration is applied on the linear FM signal, and a Chebychev window with $70 \mathrm{~dB}$ relative sidelobe attenuation is applied on the compression filter $h_{m}(t)$. The modified linear FM signal and compression filter are shown in the left and right parts of Fig. 4, respectively. The same parameters as given above have been used. The compression output is shown as the solid curve in bottom figure. As seen the temporal sidelobes have been reduced below $-60 \mathrm{~dB}$, which is adequate for clinical imaging.

\section{Signal-to-Noise-Ratio}

The (peak) SNR after matched filtering is proportional to the energy in the received signal [4]. Also, the peak compression output is proportional to the energy in the linear FM signal as seen in (8). Thus, the analysis can be simplified by only considering the energy, when comparing the SNRs obtained by linear array imaging and TMS imaging.

The noise in the system includes both analog electronics noise produced in the front-end of the system, and the quantization 
noise generated by the analog-to-digital converters (ADC) on each channel. These noise sources are assumed to be white and uncorrelated between channels. The quantization noise power is determined by the resolution of the ADC, and it is independent of the input signal and the imaging technique. Therefore, the influence of the quantization noise can be discarded under the assumption, that the input signal fills a significant part of the ADC input range, which is generally achieved in practical systems. Thus, only the analog electronics noise is considered in the analysis.

It is assumed, that the received signals from each channel are perfectly phase aligned. In this case there is no difference between transmitting with the same aperture $M$ times or with $M$ different apertures with the same size. Thus, the beamformer can be regarded as simply averaging the received channel signals. Furthermore, attenuation and diffraction effects are not included in this analysis, and it is assumed that no amplitude weighting is applied to the transmitted waveforms and receive filters. Using these assumptions a simple model for the improvement in SNR can be established. For linear array imaging, the energy in the received signal for a single element $E_{R_{L}}$ from a point target at the acoustic focus can be approximated by the number of transmit elements $N_{T_{L}}$ squared times the duration of the transmitted pulse $\tau_{l}$

$$
E_{R_{L}} \sim N_{T_{L}}^{2} \tau_{l}
$$

As shown experimentally by Karaman and coworkers [11], the amplitude of the wavefront created by the defocused subaperture consisting of $N_{T_{S}}$ elements is proportional to $\sqrt{N_{T_{S}}}$. Thus, the energy in the received signal for a single element $E_{R_{S}}$ for TMS imaging is

$$
E_{R_{S}} \sim N_{T_{S}} \tau_{s}
$$

where $\tau_{s}$ is the duration of the transmitted FM signal. As mentioned above the beamformer is considered as simply averaging the individual channel signals. Denoting the number of receive channels for linear array imaging $N_{R_{L}}$, the SNR in the linear array image is proportional to

$$
\mathrm{SNR}_{B_{L}} \sim N_{R_{L}} N_{T_{L}}^{2} \tau_{l}
$$

since the noise is white and uncorrelated between channels. From the description of STA beamforming, (3) in combination with (1) states that in TMS imaging averaging is done over $N_{R_{S}}$ receive channels and $M$ transmit events. Thus, the SNR in the TMS image is proportional to

$$
\mathrm{SNR}_{B_{S}} \sim M N_{R_{S}} N_{T_{S}} \tau_{s} .
$$

The ratio between (12) and (11) gives the improvement $I_{B}$ in SNR

$$
I_{B}=\frac{\mathrm{SNR}_{B_{S}}}{\mathrm{SNR}_{B_{L}}} \sim \frac{M N_{T_{S}}}{N_{T_{L}}^{2}} \frac{N_{R_{S}}}{N_{R_{L}}} \frac{\tau_{s}}{\tau_{l}} .
$$

This assumes that the noise is stationary and described by the same probability density function for both systems. If a better SNR is to be obtained by TMS imaging, this ratio needs to be greater than 1 .

In this study, a subaperture of $N_{T_{S}}=33$ elements will be used. The applied FM signal has a duration of $\tau_{s}=20 \mu \mathrm{s}$, and the transducer has 128 transmit and receive elements, which results in $M=96$ transmissions. Using (13) and a linear array imaging setup of $N_{T_{L}}=64, N_{R_{L}}=N_{R_{S}}=128$, and a 2 cycle sinusoid excitation at $7 \mathrm{MHz}$, it is found that $I_{B} \simeq 54.2 \approx 17.3$ $\mathrm{dB}$. This indicates that a significant increase in SNR is to be expected.

In general, the energy in the waveform received from a point target is not directly related to the duration of the transmitted pulse. Rather, it depends on both the duration and the shape. The approximation used to obtain (9) and (10) holds if the received waveform has a rectangular envelope, but under normal conditions this is not obtained due to the finite bandwidth of the transducer. Also, as mentioned previously, amplitude weighting is applied on the linear FM signal and compression filter for temporal sidelobe reduction. Therefore, the actual improvement in SNR may differ slightly from result above, because these factors have not been included in the model for simplicity. Their influence is investigated further in the next section.

\section{SimUlations}

Simulations are done using Field II [21], [22] to compare the performance of TMS imaging to linear array imaging on a theoretical basis in terms of spatial and contrast resolution.

The transducer model used is a 128-element linear array aperture with a center frequency of $8.5 \mathrm{MHz}$ and a relative bandwidth of $60 \%$. The pitch is $0.208 \mathrm{~mm}$ and the element height is $4.5 \mathrm{~mm}$. The transducer has an elevation lens with a focal point at $25 \mathrm{~mm}$. The impulse response of the receive elements is set to the pulse-echo response measured from a plane reflector in water using a delta excitation for the transducer used for the measurements in Section V. Thus, the impulse response of the transmit aperture is set to a Dirac delta function. In this way, the simulation model resembles the true situation more accurately.

For linear array imaging a 64-element aperture is used for transmission and all 128 elements are used in receive. A twocycle sinusoid at $7 \mathrm{MHz}$ weighted with a Hanning window is used as excitation signal, and no transmit apodization is applied. The received signals are filtered using a matched filter, and beamformed using dynamic receive focusing with updated delay curves for every second sample. The number of lines in the image and their spacing is calculated from the highest spatial frequency of the point spread function (PSF) to satisfy the spatial Nyquist theorem. This results in images with line counts between 132 and 165 for transmit foci at 50 and $40 \mathrm{~mm}$, respectively.

TMS imaging is done using a 33 element subaperture and 128 receive elements, which results in 96 emissions. The excitation signal is the linear FM signal displayed in Fig. 4 (top, left). It has a duration of $20 \mu \mathrm{s}$, a center frequency of $7 \mathrm{MHz}$, and a bandwidth of approximately $7 \mathrm{MHz}$. The FM signal has been weighted with a Tukey window to reduce the temporal sidelobes. The corresponding compression filter is also shown in Fig. 4 (top, right). A Chebychev window has been applied to reduce the temporal sidelobes in the compression output. The position of the virtual point source is chosen such that the distance to the subaperture is equal to the size of the subaperture. This has been determined through wavefront simulations [23]. A Tukey like window function is applied on the subaperture to reduce the influence of edge waves on the PSF. The resulting PSF is shown in Fig. 5 (top), and the corresponding PSF when 


\section{3 element defocused subaperture}

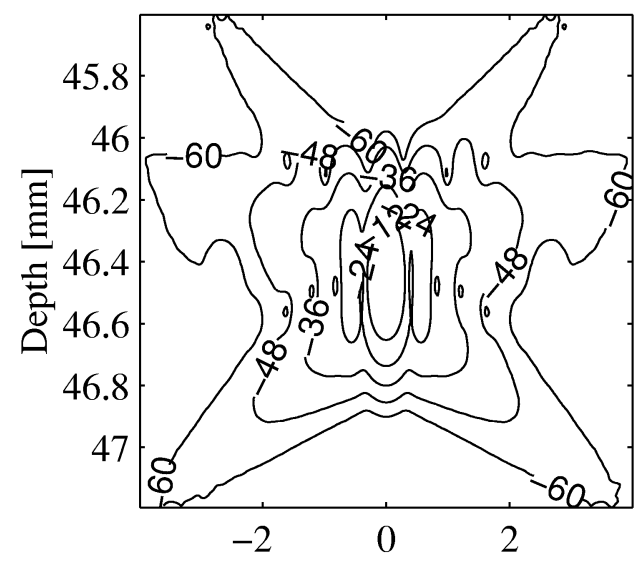

Single transmit element

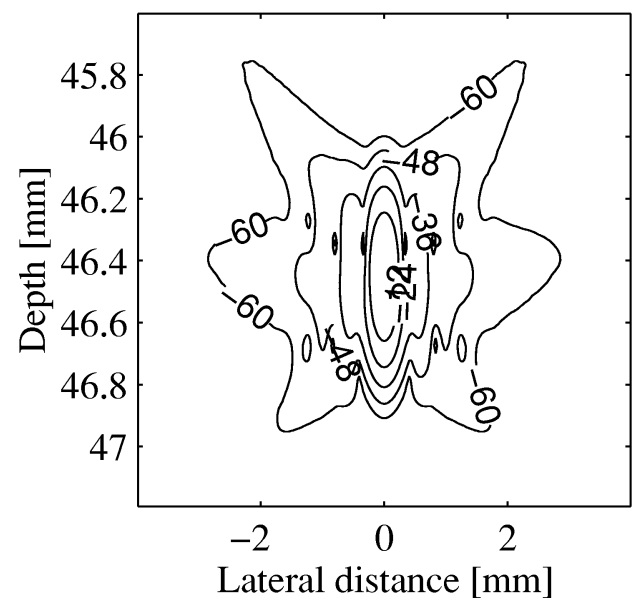

Fig. 5. PSFs obtained when using a defocused 33 element subaperture (top) and a single transmit element (bottom). The virtual point source for the multi element subaperture is placed behind the center of aperture at a distance equal to the size of the subaperture itself. The distance between the contours is $12 \mathrm{~dB}$. Notice the slightly wider multi element PSF, which indicates a slight degradation in lateral resolution compared to the single element transmission.

using a single transmit element is shown in the bottom part of the figure. As seen, the two PSFs are very similar, but the multielement PSF is a bit wider in the lateral direction, which indicates a slightly degraded lateral resolution. STA Beamforming is done with dynamic transmit and receive focusing as explained previously in Section II-A, and the received echo signals on each channel are compressed prior to beamformation. For both linear array imaging and TMS imaging, a dynamic receive aperture is used with an expanding modified Hamming window with edge levels at $-12 \mathrm{~dB}$. The aperture opens at an F-number of 2.

\section{A. Acoustic Output and SNR Simulations}

When comparing two imaging techniques it is important that both their acoustic outputs are within the official limits. The US FDA has set pre-Amendments upper limits on the acoustic outputs which may occur in the human body. These are: $I_{\text {spta.3 }} \leq$ $720 \mathrm{~mW} / \mathrm{cm}^{2}, I_{\text {sppa. }} \leq 190 \mathrm{~W} / \mathrm{cm}^{2}$, and $\mathrm{MI} \leq 1.9$ [1]. The measurement of these parameters are done following the guidelines set by the American Institute of Ultrasound in Medicine (AIUM) [2]. A simulation can, however, be done following the same guidelines to give a theoretical measure of the acoustic output. The US FDA limits are global maximum values. MI and
$I_{\text {sppa.3 }}$ are taken at the spatial location, where the derated pulse intensity integral $\left(P I I_{.3}\right)$ is maximum, while $I_{\text {spta.3 }}$ is taken at the spatial location of its global maximum [2]. For both linear array and TMS imaging this will occur on-axis of the transducer, but at different depths. For TMS imaging $P I I_{.3, \max }$ and $I_{\text {spta.3, max }}$ occur at the acoustic focus of the elevation lens at $17 \mathrm{~mm}$. For linear array imaging $P I I_{.3}$, max occurs at the lateral acoustic focus at $38 \mathrm{~mm}$ (geometric focal point at $40 \mathrm{~mm}$ ), while $I_{\text {spta.3, max }}$ occurs at the acoustic focus of the elevation lens at $17 \mathrm{~mm}$. To ease the comparison, the amplitudes of both excitation signals have been set equal and scaled such that linear array imaging reaches the $I_{\text {sppa. } 3}$ limit. In this case the obtained values are for linear array imaging: $I_{\text {sppa.3 }}=190 \mathrm{~W} / \mathrm{cm}^{2}$, $I_{\text {spta.3 }}=62.7 \mathrm{~mW} / \mathrm{cm}^{2}, \mathrm{MI}=1.07$, and for TMS imaging: $I_{\text {sppa. } 3}=35.9 \mathrm{~W} / \mathrm{cm}^{2}, I_{\text {spta. } 3}=1749.2 \mathrm{~mW} / \mathrm{cm}^{2}, \mathrm{MI}=0.45$. As seen, MI and $I_{\text {sppa.3 }}$ are lower for TMS imaging due to the linear array transmit focus, and $I_{\text {spta.3 }}$ for TMS imaging is significantly higher than linear array imaging. Compared to the US FDA limits, however, $I_{\text {spta. } 3}$ for TMS imaging is a factor of 2.4 larger than the FDA limit, while both MI and $I_{\text {sppa.3 }}$ are significantly below the limits. Since the acoustic output of a scanner must comply with all the limits, the excitation voltage for TMS imaging needs to be reduced by a factor of $\sqrt{2.4} \simeq 1.55$ compared to the linear array excitation voltage in order to satisfy the requirements. This reduction will affect all the output measures. Alternatively, the duration of the linear FM signal can be reduced by a factor of 2.4 , which will only affect $I_{\text {spta.3. }}$. It should be noted, that nonlinear effects are not included in this analysis as well as measurement noise, hydrophone effects, etc. Also, this analysis assumes that it is possible to apply excitation voltages in the scanner to reach the $I_{\text {sppa.3 }}$ FDA limit, which will depend on the front-end electronics and transducer, and, thus, the limit might not be reachable in linear array imaging. This is investigated in further detail in Section V.

It was shown in Section III-C that a significant improvement in SNR for TMS imaging is to be expected using the imaging setup described above. The model proposed model does, however, not account for the deviation from a rectangular envelope of the excitations and matched filters, and it does not include the influence of the transducer impulse response and acoustical effects, e.g., diffraction. Thus, a Field II simulation is used to take these factors into account and get a closer estimate. The excitation voltages are set equal for both methods in the analysis. If, however, the scaling of 1.55 was used, the results presented below would be reduced by $10 \log 1.55^{2} \simeq 3.81 \mathrm{~dB}$. The simulation is done by calculating the received echo response from a set of wires displaced in the axial dimension. The wires are located on-axis of the transducer at $20-90 \mathrm{~mm}$ with 5-mm spacing. The line corresponding to the position of the wires is then beamformed for both linear array imaging and TMS imaging. The ratio between the peak responses for TMS and linear array imaging is then taken for each wire, and the results are plotted in Fig. 6. The dashed curve is the result, when no amplitude weighting of the excitation signals, matched filters, and receive apertures (no fixed or dynamic apodization) are used. In this case the difference between the SNR model described in Section III-C and the simulation is the influence of the transducer and the acoustics. The linear array data are beamformed using dynamic receive focusing, and the TMS data are beamformed using dynamic transmit and receive focusing. 


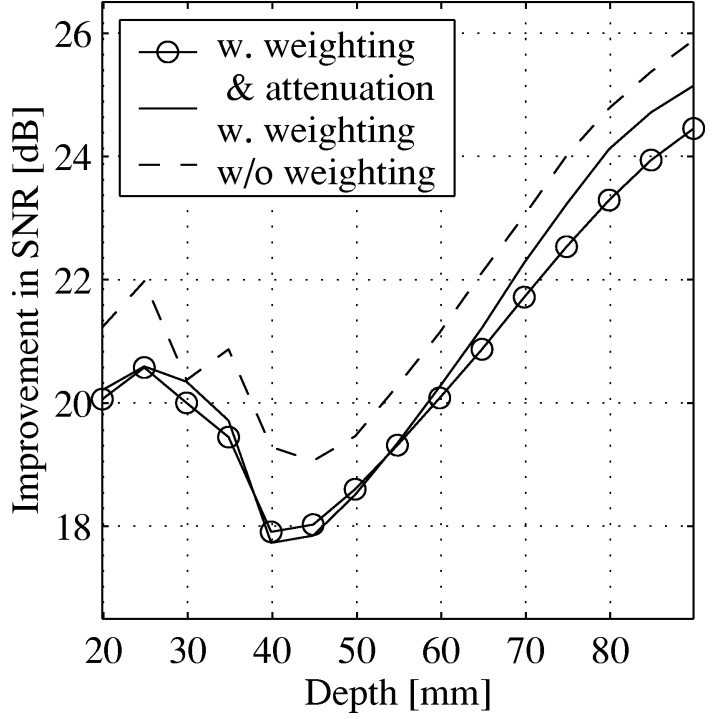

Fig. 6. Simulated SNR improvement results with (solid) and without (dashed) the application of amplitude weighting on the excitation pulses, matched filters, and the receive apertures (no dynamic aperture and expanding apodization function). The solid curve with circles is for the case when both weighting and attenuation is used. The attenuation is $0.5 \mathrm{~dB} /[\mathrm{cm} \mathrm{MHz}]$.

The improvement in SNR at $40 \mathrm{~mm}$ is $19 \mathrm{~dB}$, which is close to the $17.3 \mathrm{~dB}$ predicted by the model. This result shows that the model is useful to gain a simple understanding of the parameters, which affect the SNR. It is noted that away from the linear array focus the improvement increases, which is due to the application of dynamic transmit focusing in TMS imaging. The solid curve is the result using amplitude weighting of the excitation signals and matched filters are used, and when dynamic apodization is applied in the beamforming. At $40 \mathrm{~mm}$ the improvement in SNR has decreased to $17.7 \mathrm{~dB}$, which again is very close to the SNR model. The solid curve with circles shows the case when both weighting and attenuation is applied. The attenuation is $0.5 \mathrm{~dB} /[\mathrm{cm} \mathrm{MHz}]$. It can be seen that the attenuation does not have a significant effect until after $60 \mathrm{~mm}$, where the improvement starts to decrease compared to the case with no attenuation.

\section{B. Spatial Resolution}

To investigate the spatial resolution performance, a wire phantom is simulated with wires located at $20 \mathrm{~mm}$ to $120 \mathrm{~mm}$ with $5 \mathrm{~mm}$ spacing. The transmit focal point for linear array imaging was set to $50 \mathrm{~mm}$. Fig. 7 shows the $-6 \mathrm{~dB}$ lateral (top) and axial resolutions (bottom) as a function of depth for linear array imaging (dashed) and TMS imaging (solid), respectively. The lateral resolution is approximately constant until the aperture is fully opened at $53 \mathrm{~mm}$. Beyond this depth the lateral performance of TMS imaging is better due dynamic transmit focusing. At $120 \mathrm{~mm}$ the lateral resolution has improved by approximately $30 \%$. The performance of the axial resolutions is seen to be close, but the compression mechanism of the linear FM signal is more stable with depth.

\section{Contrast Resolution}

The contrast resolution is evaluated by simulating a cyst phantom containing sixteen anechoic cysts placed in two columns with a start depth at $30 \mathrm{~mm}$. The cysts have a diameter
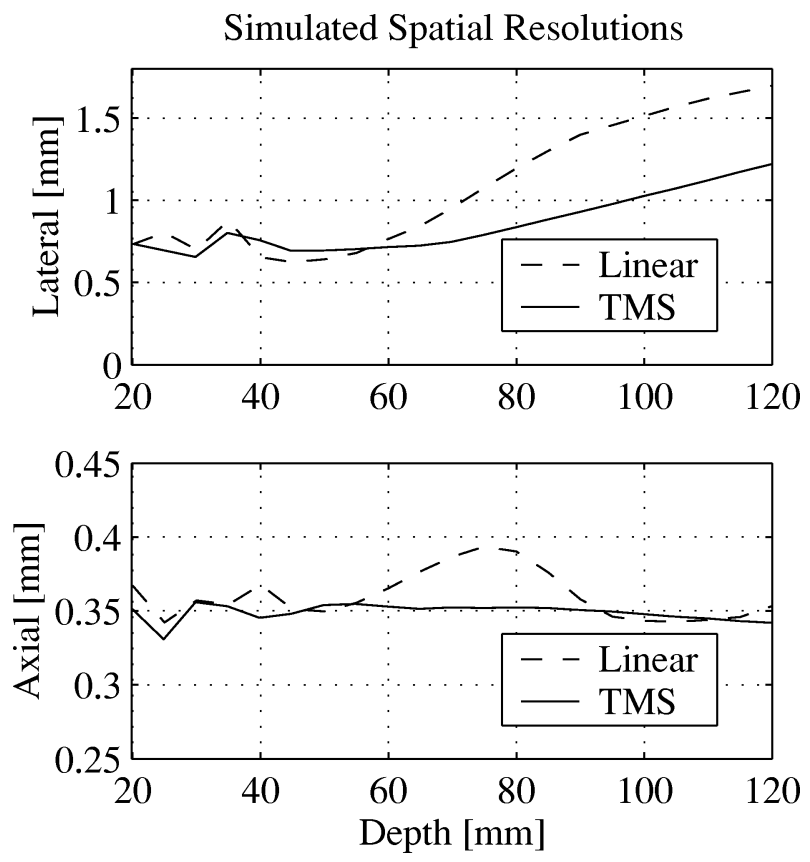

Fig. 7. (top) Lateral and (bottom) axial $(-6 \mathrm{~dB})$ resolution for linear array imaging (dashed) and TMS imaging (solid), respectively. The results are obtained from a simulated wire phantom with $5-\mathrm{mm}$ spacing between the wires. The transmit focal length for linear array imaging was set to $50 \mathrm{~mm}$.

of $4 \mathrm{~mm}$, and they are separated by $10 \mathrm{~mm}$ in depth and $5 \mathrm{~mm}$ laterally. All cysts have an amplitude of $-40 \mathrm{~dB}$ compared to the surrounding speckle. The linear array transmit focus was again set to $50 \mathrm{~mm}$, and no attenuation effects are included. The results are shown in Fig. 8. To enhance the visualization of the results each image has been divided into two parts as seen. The left part of the figure shows the results from $25 \mathrm{~mm}$ to $65 \mathrm{~mm}$, and the right part shows the results from $65 \mathrm{~mm}$ to $105 \mathrm{~mm}$. The latter clearly shows the improvement in lateral resolution for TMS imaging as described in the previous section. This has a positive effect on the contrast resolution which is improved for TMS imaging at depths beyond $70 \mathrm{~mm}$. From the images in the left part of the figure no conspicuous difference in image quality is seen.

As a measure of the contrast resolution (CR), the following equation is used:

$$
\mathrm{CR}=1-\frac{I_{c}}{I_{s}}
$$

where $I_{c}$ is the intensity inside the cyst, and $I_{s}$ is the intensity of the speckle within a region of the same size as the cyst and at the same depth. This equation gives results between zero and one, where one corresponds to perfect contrast resolution.

When calculating CR, the original size of the cyst in used and not the size of the dark region displayed in the images. This is done to take into account both the influence of the lateral resolution and the sidelobes and grating lobes. The calculated CRs are summarized in Table I. For each imaging method the numbers have been averaged for each depth. In general, these numbers show an overall improvement in contrast resolution for TMS imaging. The lowest improvement is about $1 \%$ and it is obtained for the cyst at $60 \mathrm{~mm}$. This is also the first cyst for which the receive aperture is fully opened. Beyond this depth the contrast resolution improves and at $100 \mathrm{~mm}$ the improvement is $10.75 \%$. 

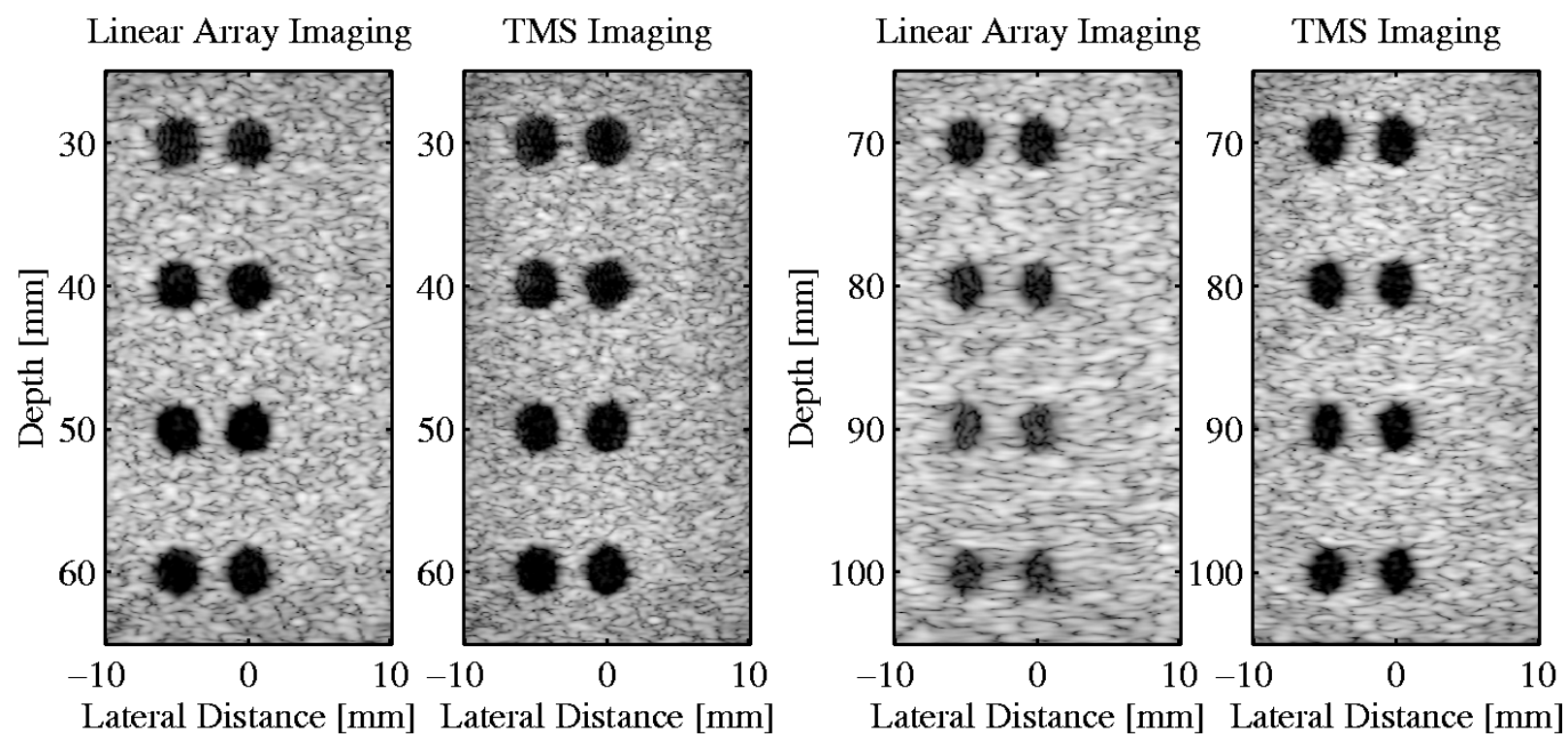

Fig. 8. Images of the simulated cyst phantom. The images have been divided into two parts with the region $25 \mathrm{~mm}$ to $65 \mathrm{~mm}$ displayed in the left part of the figure, and the region $65 \mathrm{~mm}$ to $105 \mathrm{~mm}$ is shown to the right. In both parts of the figure, the linear array image is shown to the left and the TMS image to the right. No attenuation effects are included in the simulation.

TABLE I

CALCUlated CONTRASTS FOR THE Simulated CYst Phantoms

\begin{tabular}{c|c||c|c|c}
\hline Cyst & Depth & Linear Array Imaging & TMS Imaging & Improvement in \% \\
\hline 1 & $30 \mathrm{~mm}$ & 0.92 & 0.98 & 5.64 \\
\hline 2 & $40 \mathrm{~mm}$ & 0.91 & 0.95 & 3.75 \\
\hline 3 & $50 \mathrm{~mm}$ & 0.94 & 0.97 & 2.77 \\
\hline 4 & $60 \mathrm{~mm}$ & 0.92 & 0.92 & 1.06 \\
\hline 5 & $70 \mathrm{~mm}$ & 0.94 & 0.96 & 2.30 \\
\hline 6 & $80 \mathrm{~mm}$ & 0.88 & 0.95 & 7.57 \\
\hline 7 & $90 \mathrm{~mm}$ & 0.86 & 0.95 & 10.31 \\
\hline 8 & $100 \mathrm{~mm}$ & 0.86 & 0.95 & 10.75 \\
\hline
\end{tabular}

Above $60 \mathrm{~mm}$ the contrast resolution has also improved. This is also expected due to the dynamic transmit focusing.

\section{MEASUREMENTS}

The measurements are performed using the experimental multichannel ultrasound scanning system, RASMUS [13]. The system has 128 individually programmable transmitters capable of sending arbitrary coded waveforms with a precision of 12 bits at $40 \mathrm{MHz}$. Sixty-four receive channels can be simultaneously sampled at 12 bits and $40 \mathrm{MHz}$, and the 2 to 1 multiplexing in the system enables acquisition of 128 channels in real-time over two transmissions. The RASMUS system is remotely accessible and programmed through a developed Matlab/C-library interface. The system is build using a slightly modified commercial front-end, which is limited to a supply voltage of $\pm 100 \mathrm{~V}$. In practice, however, $\pm 50 \mathrm{~V}$ or $100 \mathrm{Vpp}$ is used. Thus, the system represents a commercially available scanner.

The transducer used for the measurements has the same parameters as the transducer used for the simulations. Also, the measurement setups for linear array imaging and TMS imaging are the same as those used for the simulations. This has been chosen to enable direct comparison between the measurements and the simulations. The filters used for matched filtering and compression of the received signals have been normalized equally for both methods to enable a fair comparison, and the beamforming of the measured data is also done as for the simulated data.

\section{A. Acoustic Output Measurements}

According to the acoustic output simulations presented in Section IV, the excitation voltage used in TMS imaging needs to be reduced a factor of 1.55 compared to the linear array excitation voltage to comply with the FDA limits. This analysis assumed that the excitation voltage could be increased to reach the $I_{\text {sppa.3 }}$ FDA limit. However, in the RASMUS system, this might not be achievable due to limitations of the front-end electronics and heating of the transducer. Also, the simulations did not account for several practical effects, such as the nonlinear effects, the influence of the hydrophone, noise, etc., which also will influence the output quantities. Therefore, the acoustic output should be measured to obtain a direct relation to the FDA requirements.

Due to current limitations of our measurement setup only MI and $I_{\text {sppa.3 }}$ have been measured. The measurements were done in a water tank according to the guidelines in [2] for both linear array and TMS imaging. $P I I_{.3, \max }$ was found on-axis for both methods, but at $17 \mathrm{~mm}$ for TMS imaging and at $40 \mathrm{~mm}$ for linear array imaging (geometric focal point at $40 \mathrm{~mm}$ ). For linear array imaging the measured quantities were: $\mathrm{MI}=0.12$ and $I_{\text {sppa. } 3}=10.0 \mathrm{~W} / \mathrm{cm}^{2}$, and TMS imaging: $\mathrm{MI}=0.10$ and $I_{\text {sppa. } 3}=1.94 \mathrm{~W} / \mathrm{cm}^{2}$. As seen all quantities are significantly below the FDA limits. In particular, $I_{\text {sppa.3 }}$ for linear array imaging is 19 times less than the FDA limit. Thus, the excitation voltage in the RASMUS system can be increased $\sqrt{19} \simeq 4.4$ times for linear array imaging, assuming a linear relation between the excitation voltage and the pressure, to reach 


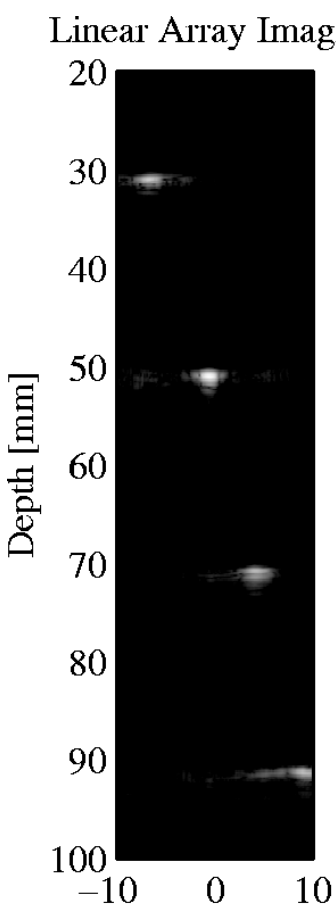

Lateral Distance [mm]
Axial Projection

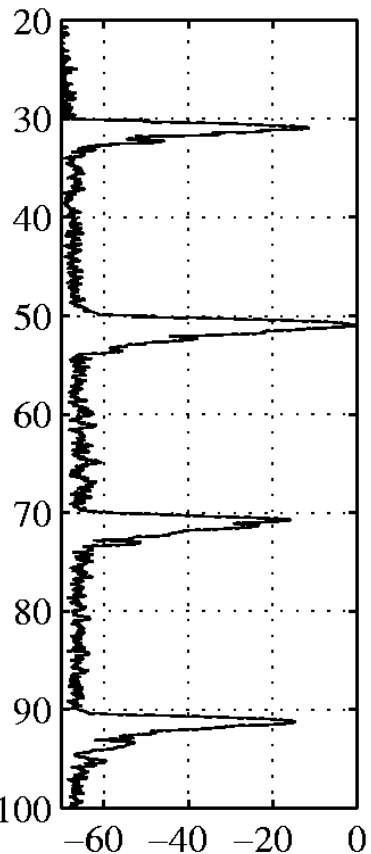

Amplitude [dB]
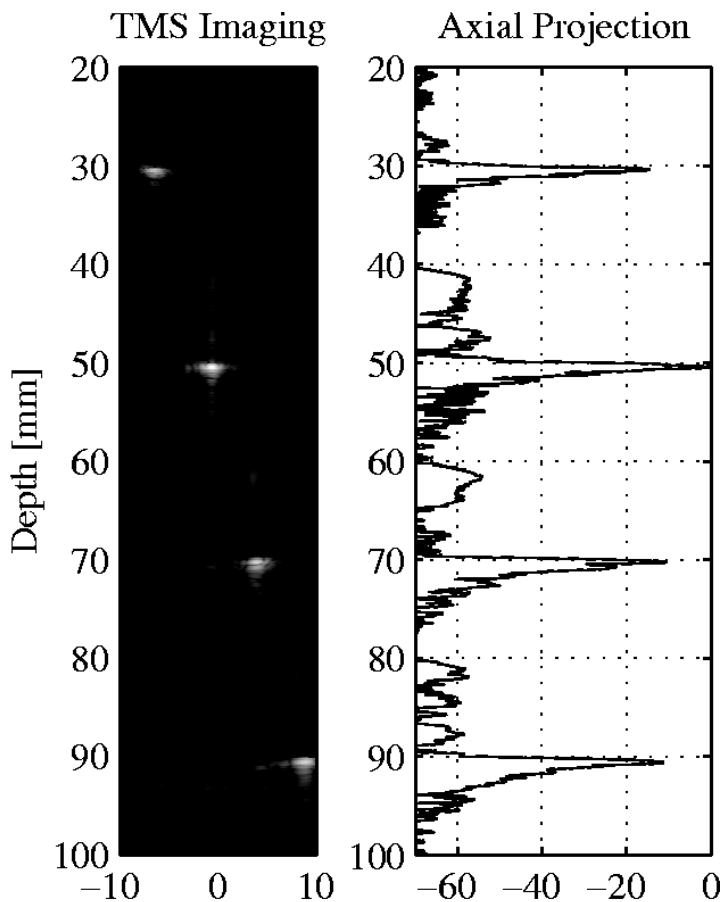

Lateral Distance [mm] Amplitude [dB]

Fig. 9. Measured B-mode images and axial projections of a scanned wire phantom using linear array imaging (left) and TMS imaging (right). The phantom contains four laterally and axially spaced copper wires and water, and the dynamic range in the B-mode image is $60 \mathrm{~dB}$. As seen in the projection image for TMS imaging, the applied linear FM signal and compression filter yields temporal sidelobes below $-55 \mathrm{~dB}$.

the FDA limit. This results in an excitation voltage of approximately $\pm 220 \mathrm{~V}$, which significantly exceeds the limitations of the front-end and the transducer. The companion excitation voltage for TMS imaging would be $( \pm 220 \mathrm{~V}) / 1.55 \simeq \pm 142$ $\mathrm{V}$, which likewise exceeds the limitation in the front-end. If the excitation voltage is increased to $\pm 100 \mathrm{~V}$, the acoustic output for TMS imaging will still be below the FDA limit. Keeping the excitation voltages equal for both methods, therefore, does not violate the basis for a fair comparison. Thus, the excitation voltage is $\pm 50 \mathrm{~V}$ for all measurements. It should be mentioned, however, that if the power supply voltage could be increased beyond $\pm 142 \mathrm{~V}$, the excitation voltages would have to be different for TMS and linear array imaging to comply with the FDA regulations and to make the comparison fair.

\section{B. Phantom Measurements}

The linear FM signal used in both the simulations and measurements has been designed to obtain temporal sidelobe levels at approximately $-60 \mathrm{~dB}$. To evaluate this, a wire phantom containing four wires in water has been scanned. Fig. 9 shows the B-mode images and axial projections for linear array imaging (left) and TMS imaging (right). The axial projections are obtained by taking the maximum at each depth in the images. The dynamic range in the B-mode images is $60 \mathrm{~dB}$. As seen the temporal sidelobes have been reduced to approximately $-55 \mathrm{~dB}$, which is adequate for clinical imaging. Also, it is noticed, that the lateral sidelobe levels are lower both above and below the linear array transmit focus. This observation is inspected more closely in Fig. 10, which shows the lateral projections of the PSF at depth $70 \mathrm{~mm}$ in both B-mode images. The lateral projection

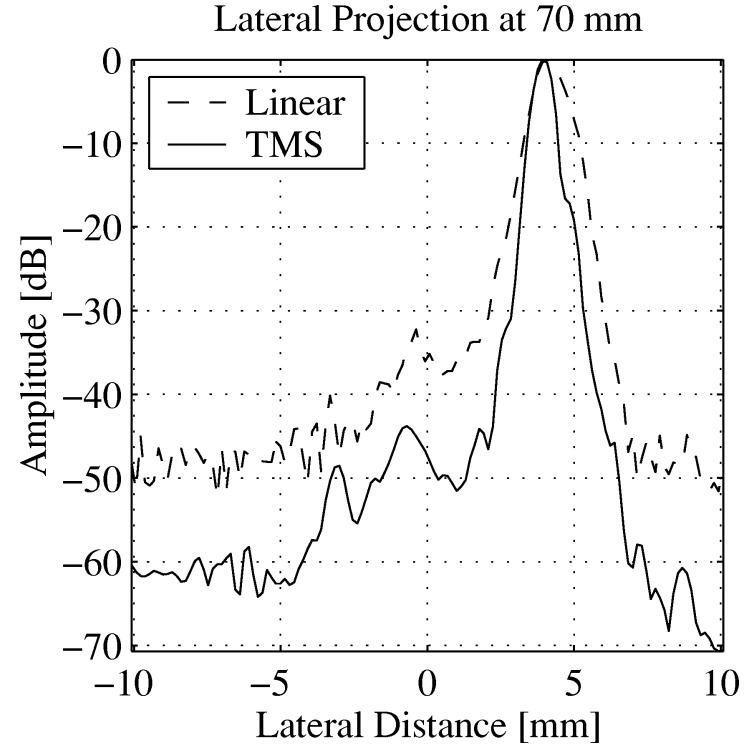

Fig. 10. Lateral projection of the PSFs at depth $70 \mathrm{~mm}$ in Fig. 9. The dashed line is linear array imaging and the solid line is TMS imaging.

is obtained by taking the maximum of each line within the region containing the PSF. The reduction in sidelobe levels is up to $15 \mathrm{~dB}$.

The spatial resolution and SNR performance of TMS imaging is evaluated in the presence of attenuation using a multitarget phantom with $0.5 \mathrm{~dB} /[\mathrm{cm} \mathrm{MHz}]$ attenuation. Fig. 11 shows the linear array image (left) and TMS image (right) of a scanned region containing twisted nylon wires spaced axially by $1 \mathrm{~cm}$. The dynamic range is $50 \mathrm{~dB}$ in both images. The penetration depth has been increased roughly $3 \mathrm{~cm}$ using TMS imaging. 


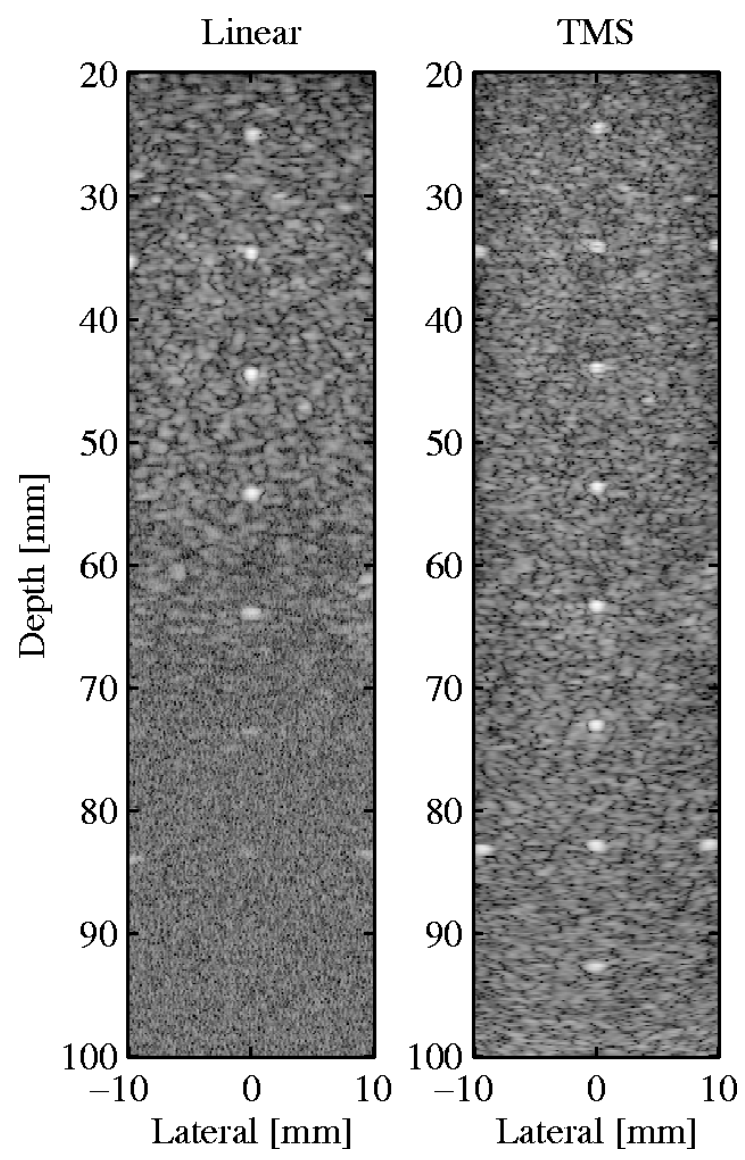

Fig. 11. Measured linear array image (left) and TMS image (right) on a multitarget phantom with $0.5 \mathrm{~dB} /[\mathrm{cm} \mathrm{MHz}]$ attenuation. The scanned section contains twisted nylon wires spaced axially by $1 \mathrm{~cm}$ throughout the imaged region. The dynamic range in the images is $50 \mathrm{~dB}$.

The linear array image has a very low SNR after $70 \mathrm{~mm}$, and the wires are not visible in this region. No apparent noise is present in the TMS image, and the wires are, thus, fully visible throughout the imaged region.

The $-6 \mathrm{~dB}$ lateral and axial resolutions are evaluated for each wire in both images in Fig. 11. The results are shown in Fig. 12, where the lateral resolution is displayed in the top figure and the axial resolution in the bottom figure. TMS imaging has a better lateral performance at distances after the linear array transmit focus due to dynamic transmit focusing. Compared to the simulation results in Fig. 7, it is noted that the lateral resolutions follow the same trends, but it is a bit higher in the measurements due to the presence of attenuation. The axial resolution is better for TMS imaging throughout the imaged region. This shows that the linear FM signal has a better axial performance in the presence of attenuation than the conventional short excitation pulse. The axial resolution for linear array imaging can, however, be improved by applying a shorter excitation pulse instead of the two cycles pulse used in this study. This will though result in less transmitted energy and, thus, lower penetration and SNR.

For the water SNR the linear array transmit focus was set to 50 $\mathrm{mm}$, and at this depth the SNR improvement is $8 \mathrm{~dB}$. This result does not compare exactly to the SNR model proposed earlier and the simulations, which showed an improvement of approximately $18 \mathrm{~dB}$. There are, however, many factors which cannot be included in a model and in the simulations. One of these factors
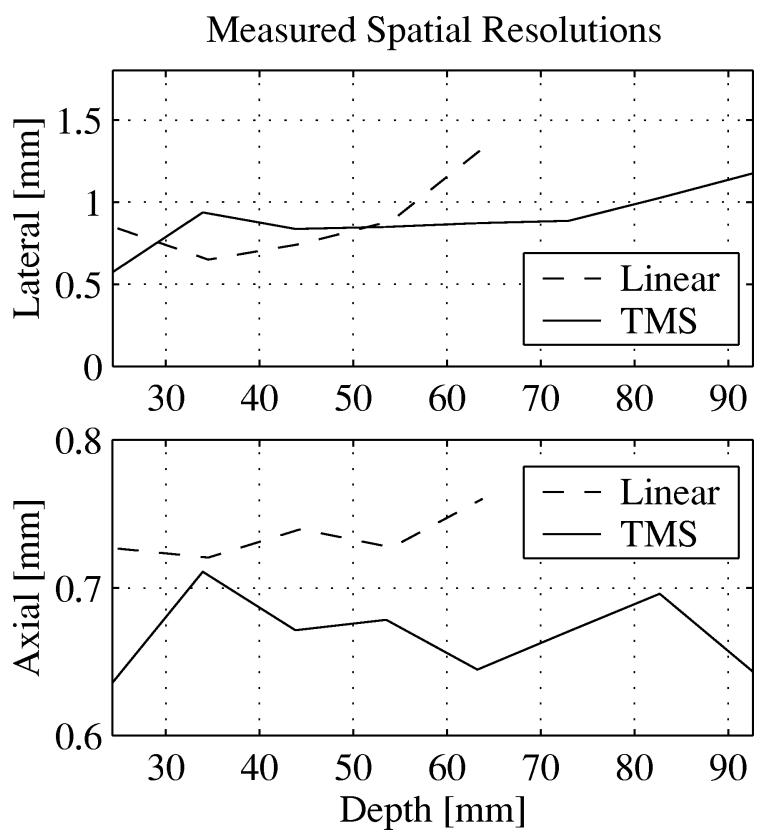

Fig. 12. Lateral (top) and axial (bottom) resolution curves for linear array imaging (dashed) and TMS imaging (solid). The curves have been obtained by calculating the spatial resolutions for each wire in Fig. 11.

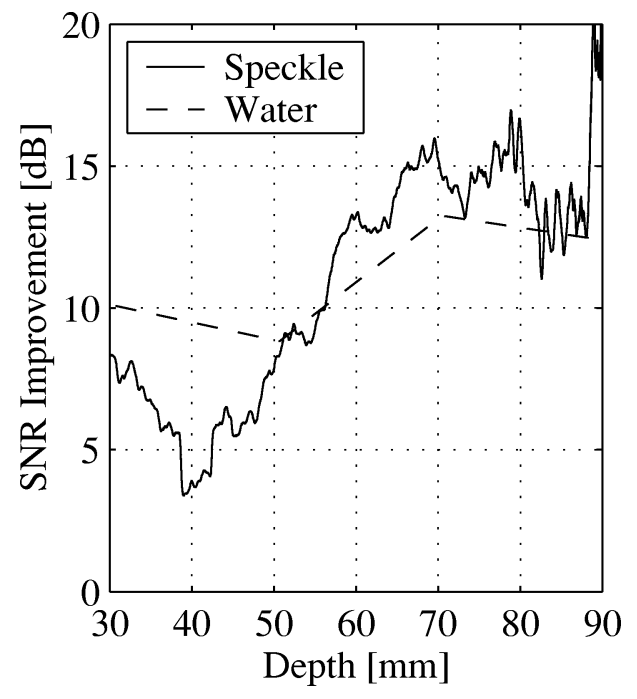

Fig. 13. Calculated SNR improvement obtained by TMS imaging in water (dashed) and in a tissue mimicking phantom (solid).

is inefficiency of the measurement system. Therefore, the deviation between the simulation and the measurement result should be looked upon as a place of possible improvement of TMS imaging. The improvement in SNR obtained by TMS imaging in water and for a tissue mimicking phantom is shown in Fig. 13. The SNR for water was obtained using the wire phantom data from Fig. 9. For each image and depth the power was calculated resulting in two signal power vectors. The noise power in the system generated by each imaging method was obtained by scanning a water bath with no reflections. For each imaging method the power of the resulting noise images was calculated, resulting in a noise power vector. Taking the ratio between the signal power vectors and the noise power vectors yields the SNR for each imaging method, and the difference between these SNR curves at the wire locations is the SNR improvement shown as 

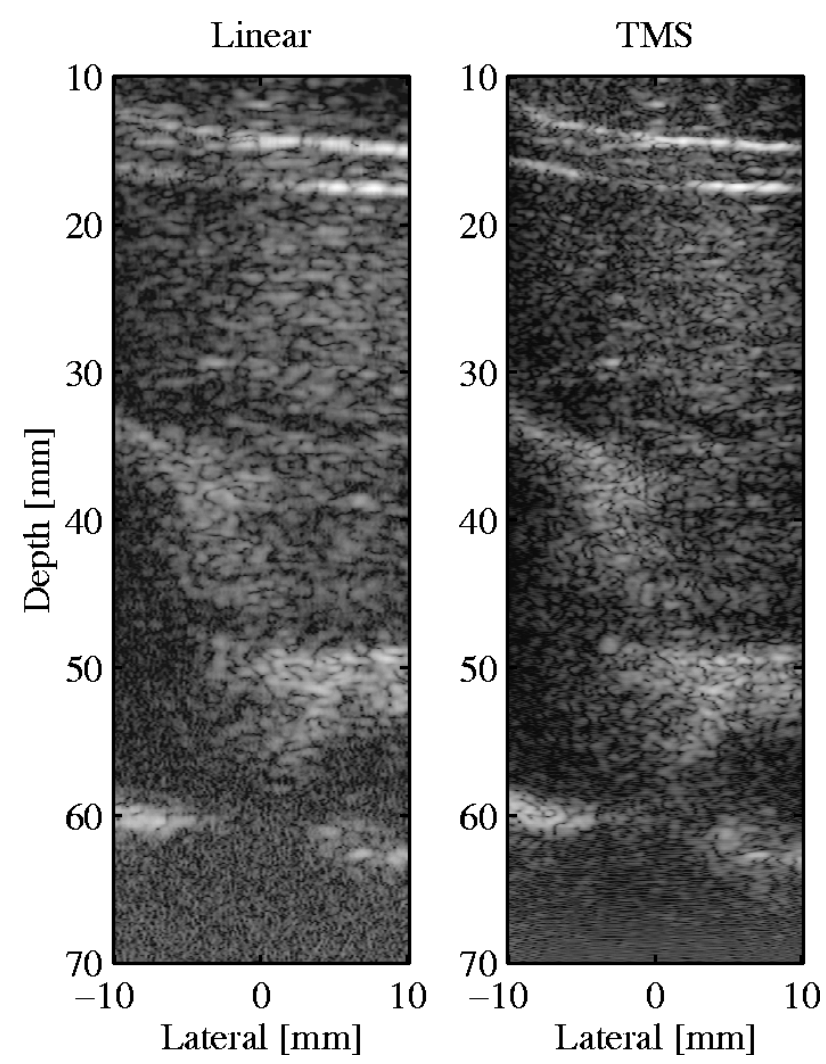

Fig. 14. In-vivo images of the abdomen using linear array imaging (left) and TMS imaging (right). The dynamic range in the image is $50 \mathrm{~dB}$

the dashed curve in Fig. 13. Since only one image was used for calculating the signal power vector, this vector contains noise. Therefore, the noise power was subtracted from the signal power vector before taking the ratio. Thus, for a given depth

$$
\mathrm{SNR}=\frac{P_{\text {signal }}-P_{\text {noise }}}{P_{\text {noise }}}
$$

where $P_{\text {signal }}$ is the signal power, and $P_{\text {noise }}$ is the noise power. The SNR improvement in the tissue mimicking phantom was obtained using the parts of Fig. 11 not containing wires and the generated noise vectors from the water bath. The SNR improvement was evaluated at each depth throughout the image, and the result is shown as the solid curve in Fig. 13. A low-pass filter has been applied on the curve to make it more smooth.

At the linear array focal point in the phantom $(40 \mathrm{~mm})$ the improvement in SNR is about $4 \mathrm{~dB}$, but it is significantly larger before and after the focus. At $80 \mathrm{~mm}$, an improvement of 15 $\mathrm{dB}$ is obtained and at approximately $90 \mathrm{~mm}$, the SNR of linear array imaging is so low that the correct values can no longer be obtained. In general, the SNR follows the same trend as the simulations, but the curve has been shifted downwards.

\section{In-Vivo Measurements}

To demonstrate the clinical feasibility of TMS imaging, in vivo measurements on the abdomen of a 27 -year-old male were performed. The measurement setups for both linear array imaging and TMS imaging are the same as those used for the phantom measurements with the linear array transmit focal depth set to $50 \mathrm{~mm}$. The RASMUS system is setup in an interleaved mode such that the linear array and TMS scannings
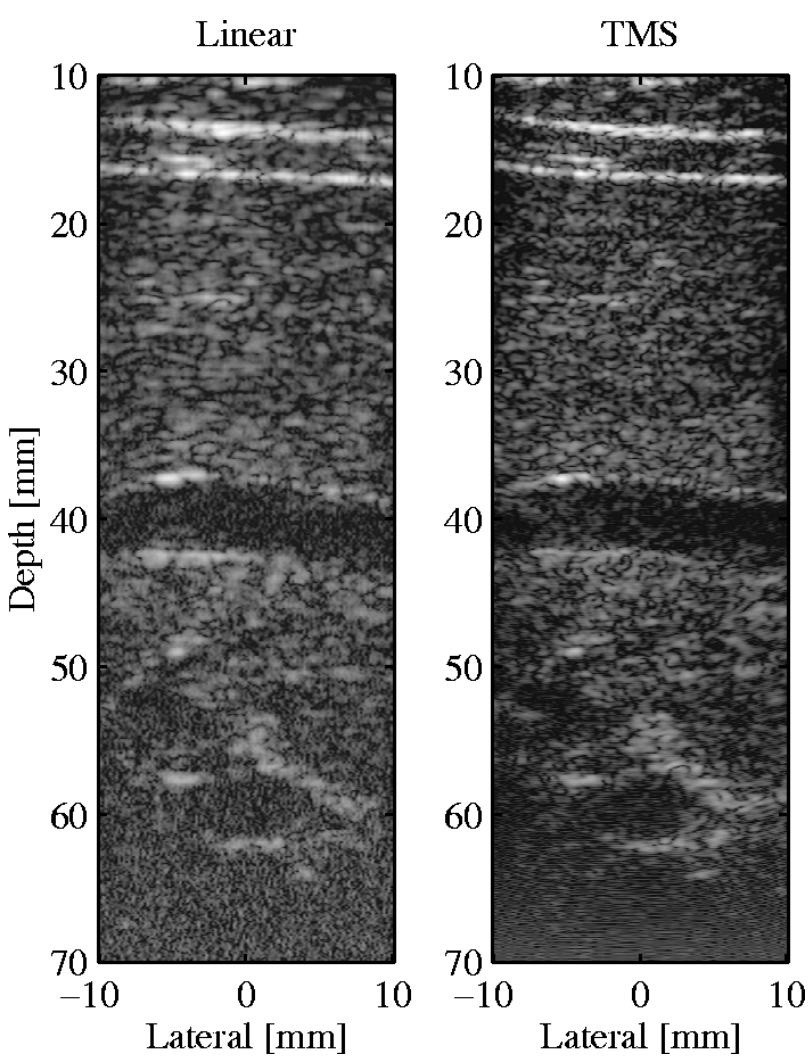

Fig. 15. Same as Fig. 14 but of a different abdominal location. The dynamic range in the image is $40 \mathrm{~dB}$.

are performed in one long sequence. Thus, data from exactly the same abdominal position are obtained at the same time, which enables direct comparison of the images. Figs. 14 and 15 show the results of two scannings at different abdominal locations. The dynamic range is $50 \mathrm{~dB}$ in Fig. 14 and $40 \mathrm{~dB}$ in Fig. 15, and the linear array and TMS images are shown to the left and right, respectively. The TMS images have both better spatial resolution and contrast resolution.

When comparing the linear array and TMS images there are no visible motion artifacts. This may seem surprising since TMS imaging in theory is more sensitive to tissue motion, because a complete image is formed at each transmission event and the images are summed in the end as explained in Section II. As reported by Trahey and Nock [24], axial tissue motion is the dominant factor in image quality degradation. Thus, in the following example only axial motion is considered. Using a pulse repetition frequency $\left(f_{p r f}\right)$ of $5 \mathrm{kHz}$, the TMS imaging system described here will have a frame rate $F R$ of approximately 25 frames/s, due to the 96 emissions and the 2-to-1 multiplexing. Schlaikjer and coworkers [25] have reported measured tissue velocities around the Hepatic vein of $10 \mathrm{~mm} / \mathrm{s}$ over a cardiac cycle. Using this velocity as the axial velocity $v_{z}$ of a tissue region, the total displacement $\Delta z$ of the tissue from the first to the last transmission, i.e., the whole frame, is

$$
\Delta z=\frac{v_{z}}{F R}=\frac{10 \mathrm{~mm} / \mathrm{s}}{25 \mathrm{frames} / \mathrm{s}}=400 \mu \mathrm{m} / \text { frame. }
$$

This is compared to an acoustic wavelength $\lambda$ of $220 \mu \mathrm{m}$, assuming a sound speed of $1540 \mathrm{~m} / \mathrm{s}$ and a center frequency of 7 $\mathrm{MHz}$. Thus, the tissue moves approximately $2 \lambda$ during the acquisition of a full high resolution TMS image. This motion will 
occur gradually over the whole acquisition, if the velocity is assumed constant. The displacement between two low resolution images is, therefore, about $4 \mu \mathrm{m}$, which is less than $2 \%$ of the wavelength, and the correlation between the images is, therefore, high. Also, the axial resolution is observed to be approximately $3 \lambda$ in Fig. 12. Therefore, it can be concluded that tissue motion in this case will not have a significant influence on the image quality of the TMS image, which is also supported by the abdominal in vivo images, that show no visual motion artifacts.

\section{CONCLUSION}

In this paper a new approach to increase the SNR of STA imaging has been investigated. The approach combines multielement STA imaging with the application of linear FM signals, and it is called TMS imaging. Several simulations using Field II and phantom and in vivo measurements were performed to investigate the performance of TMS imaging compared to linear array imaging. The overall performance of TMS imaging is better than linear array imaging. The simulations showed an improvement in lateral resolution of up to $30 \%$ at a depth of $120 \mathrm{~mm}$, and general improvements in contrast resolution of up to approximately $11 \%$ were obtained. The phantom measurements showed an increase in penetration depth of $3 \mathrm{~cm}$ on a tissue-mimicking phantom with $0.5 \mathrm{~dB} /[\mathrm{cm} \mathrm{MHz}]$ attenuation. This corresponds to an increase of approximately $45 \%$. The corresponding increase in SNR was 4-12 dB. The in vivo measurements of the abdomen showed better contrast and spatial resolution for TMS imaging and no visual distortion of the images due to tissue motion.

This paper has shown that the SNR of STA imaging can be increased to exceed that obtained by linear array imaging. The result is images with overall better image quality obtained using fewer transmission events than linear array imaging.

\section{REFERENCES}

[1] "Information for manufacturers seeking marketing clearance of diagnostic ultrasound systems and transducers," United States Food and Drug Administration (FDA) and Center for Devices and Radiological Health, Tech. Rep., 1997.

[2] "Acoustic output measurement standard for diagnostic ultrasound equipment," Amer. Inst. Ultrasound in Medicine and Nat. Electrical Manufacturers Assoc. (AIUM), Tech. Rep., May 1998.

[3] J. G. Abbott, "Rationale and derivation of MI and TI-A review," $U l$ trasound Med. Biol., vol. 25, pp. 431-441, 1999.

[4] H. J. Blinchikoff and A. I. Zverev, Filtering in the Time and Frequency Domains. New York: Wiley, 1976.

[5] D. R. Wehner, High Resolution Radar, 2nd ed. Norwood, MA: Artech House, 1996.
[6] Y. Takeuchi, "An investigation of a spread energy method for medical ultrasound systems-Pt. I: Theory and investigations," Ultrasonics, pp. 175-182, 1979.

[7] M. O'Donnell, "Coded excitation system for improving the penetration of real-time phased-array imaging systems," IEEE Trans. Ultrason., Ferroelect., Freq. Contr., vol. 39, pp. 341-351, May 1992.

[8] T. X. Misaridis, K. Gammelmark, C. H. Jørgensen, N. Lindberg, A. H. Thomsen, M. H. Pedersen, and J. A. Jensen, "Potential of coded excitation in medical ultrasound imaging," Ultrasonics, vol. 38, pp. 183-189, 2000.

[9] T. X. Misaridis, M. H. Pedersen, and J. A. Jensen, "Clinical use and evaluation of coded excitation in B-mode images," in Proc. IEEE Ultrason. Symp., vol. 2, 2000, pp. 1689-1693.

[10] T. Misaridis, "Ultrasound imaging using coded signals," Ph.D. dissertation, Ørsted • DTU, Tech. Univ. Denmark, Lyngby, Denmark, 2001.

[11] M. Karaman, P. C. Li, and M. O'Donnell, "Synthetic aperture imaging for small scale systems," IEEE Trans. Ultrason., Ferroelect., Freq. Contr., vol. 42, pp. 429-442, May 1995.

[12] S. I. Nikolov, K. Gammelmark, and J. A. Jensen, "Recursive ultrasound imaging," in Proc. IEEE Ultrason. Symp., vol. 2, 1999, pp. 1621-1625.

[13] J. A. Jensen, O. Holm, L. J. Jensen, H. Bendsen, H. M. Pedersen, K. Salomonsen, J. Hansen, and S. Nikolov, "Experimental ultrasound system for real-time synthetic imaging," in Proc. IEEE Ultrason. Symp., vol. 2, 1999, pp. 1595-1599.

[14] S. Holm and K. Kristoffersen, "Analysis of worst-case phase quantization sidelobes in focused beamforming," IEEE Trans. Ultrason., Ferroelect., Freq. Contr., vol. 39, pp. 593-599, Sept. 1992.

[15] M. O'Donnell, W. E. Engeler, J. T. Pedicone, A. M. Itani, S. E. Noujaim, R. J. Dunki-Jacobs, W. M. Leue, C. L. Chalek, L. S. Smith, J. E. Piel, R. L. Harris, K. B. Welles, and W. L. Hinrichs, "Real-time phased array imaging using digital beam forming and autonomous channel control," in Proc. IEEE Ultrason. Symp., 1990, pp. 1499-1502.

[16] G. R. Lockwood, J. R. Talman, and S. S. Brunke, "Real-time 3-D ultrasound imaging using sparse synthetic aperture beamforming," IEEE Trans. Ultrason., Ferroelect., Freq. Contr., vol. 45, pp. 980-988, July 1998.

[17] P. Li, E. Ebbini, and M. O'Donnell, “A new filter design technique for coded excitation systems," IEEE Trans. Ultrason., Ferroelect., Freq. Contr., vol. 39, pp. 693-699, Nov 1992.

[18] J. Shen and E. S. Ebbini, "A new coded-excitation ultrasound imaging system-Pt. I: Basic principles," IEEE Trans. Ultrason., Ferroelect. Freq. Contr., vol. 43, pp. 131-140, Jan. 1996.

[19] T. X. Misaridis and J. A. Jensen, "An effective coded excitation scheme based on a predistorted FM signal and an optimized digital filter," in Proc. IEEE Ultrason. Symp., vol. 2, 1999, pp. 1589-1593.

[20] Y. Takeuchi, "Chirped excitation for $<-100 \mathrm{~dB}$ time sidelobe echo sounding," in Proc. IEEE Ultrason. Symp., 1995, pp. 1309-1314

[21] J. A. Jensen and N. B. Svendsen, "Calculation of pressure fields from arbitrarily shaped, apodized, and excited ultrasound transducers," IEEE Trans. Ultrason., Ferroelect., Freq. Contr., vol. 39, pp. 262-267, Mar. 1992.

[22] J. A. Jensen, "Field: A program for simulating ultrasound systems," in Med. Biol. Eng. Comp., vol. 4, Suppl. 1, Pt. 1, vol. 10th Nordic-Baltic Conference on Biomedical Imaging, 1996b, pp. 351-353.

[23] K. Gammelmark, "Multi-element synthetic transmit aperture imaging using temporal encoding," M.S. thesis, Ørsted • DTU, Tech. Univ. Denmark, Lyngby, Denmark, 2001.

[24] G. E. Trahey and L. F. Nock, "Synthetic receive aperture imaging with phase correction for motion and for tissue inhomogenities-Pt. II: Effects of and correction for motion," IEEE Trans. Ultrason., Ferroelec., Freq. Contr., vol. 39, pp. 496-501, July 1992.

[25] M. Schlaikjer, S. Torp-Pedersen, J. A. Jensen, and P. F. Stetson, "Tissue motion in blood velocity estimation and its simulation," in Proc. IEEE Ultrason. Symp., 1998, pp. 1495-1499. 